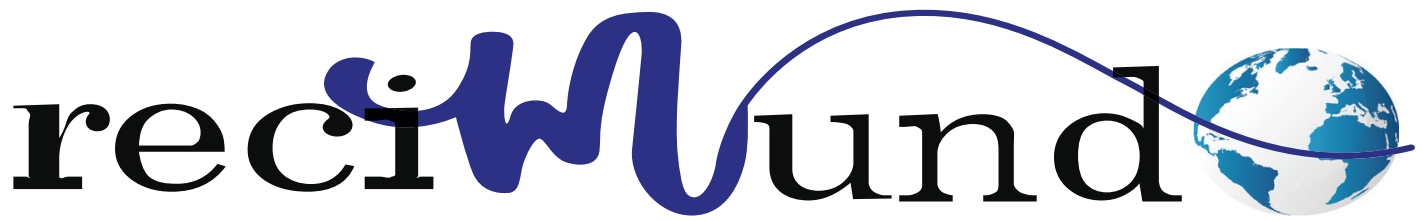

Revista Científica Mundo de la Investigación y el Conocimiento

DOI: 10.26820/recimundo/4.(1).enero.2020.71-83

URL: http://recimundo.com/index.php/es/article/view/750

EDITORIAL: Saberes del Conocimiento

REVISTA: RECIMUNDO

ISSN: 2588-073X

TIPO DE INVESTIGACIÓN: Artículo de Revisión

CóDIGO UNESCO: 3205 Medicina Interna

PAGINAS: $71-83$

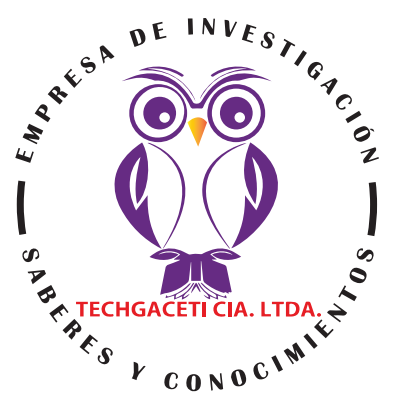

\title{
Agentes antiagregantes en los síndromes coronarios agudos
} Antiplatelet agents in acute coronary syndromes

\section{Complicações na cirurgia de hérnia de virilha}

Jose Antonio Salinas Romero1; Yulisa Veronica Arana Alcivar2; Dámarys Karina Sánchez León³;

Adriana Ximena Guachi Criollo ${ }^{4}$

RECIBIDO: 18/12/2018 ACEPTADO: 29/03/2019 PUBLICADO: 01/12/2019

1. Médico; Investigador Independiente; Guayaquil, Ecuador; ppantonio0588@hotmail.com; (D) https://orcid. org/0000-0002-0228-7661

2. Médico; Investigador Independiente; Guayaquil, Ecuador; yulita1387@hotmail.com; (D) https://orcid.org/00000001-5368-6454

3. Médico; Investigador Independiente; Guayaquil, Ecuador, Ecuador; damaryssanchez|@hotmail.com; (DD https://orcid.org/0000-0001-6237-3396

4. Médico; Investigador Independiente; Guayaquil, Ecuador; adrianaxgc@gmail.com (DD https://orcid.org/00000001-7413-5926

CORRESPONDENCIA

Sara Alexandra Carbo Yagual

sary_17_85_@hotmail.com

Guayaqui, Ecuador

○ RECIMUNDO; Editorial Saberes del Conocimiento, 2020 


\title{
RESUMEN
}

En condiciones de equilibrado, la hemostasia es mantenido por una compleja interacción entre factores endotelio, plaquetas y de la coagulación. Las situaciones que implican lesión y la interrupción del revestimiento endotelial estimulan la adhesión, activación y agregación de las plaquetas, que culmina en la formación de trombos arteriales o venosas. En este contexto, el tratamiento antiplaquetario ocupa un papel destacado en la gestión de patologías resultantes de este proceso, notablemente aguda síndromes coronarios. El aumento de la comprensión conceptual de los receptores, agonistas, y antagonistas de las cascadas fisiopatológicos involucrados en este proceso ha permitido el desarrollo de nuevos fármacos y refinamiento de la terapia actual, exigiendo una completa, conocimiento del arsenal de agentes antiplaquetarios con respecto a su indicación, dosis, momento de la administración, y la duración del tratamiento. El objetivo de esta revisión es para definir el papel de los fármacos antiplaquetarios en la gestión de síndrome coronario agudo, revisando los aspectos que han sido ya consolidados y que abordan temas de actualidad y todavía controvertidos sobre el tema.

Palabras clave: El síndrome coronario agudo; Infarto de miocardio; La agregación plaquetaria; Inhibidores de plaquetas de la sangre; Agentes antifibrinolíticos.

\begin{abstract}
Under balanced conditions, hemostasis is maintained by a complex interaction between endothelial, platelet and coagulation factors. Situations that involve injury and disruption of the endothelial lining stimulate the adhesion, activation and aggregation of platelets, which culminates in the formation of arterial or venous thrombi. In this context, antiplatelet treatment occupies a prominent role in the management of pathologies resulting from this process, notably acute coronary syndromes. The increase in the conceptual understanding of the receptors, agonists, and antagonists of the pathophysiological cascades involved in this process has allowed the development of new drugs and refinement of the current therapy, demanding a complete, knowledge of the arsenal of antiplatelet agents with respect to their indication, dose, time of administration, and duration of treatment. The objective of this review is to define the role of antiplatelet drugs in the management of acute coronary syndrome, reviewing aspects that have already been consolidated and that address current and still controversial issues on the subject.
\end{abstract}

Keywords: Acute coronary syndrome; Myocardial infarction; Platelet aggregation; Blood platelet inhibitors; Antifibrinolytic agents.

\section{RESUMO}

Sob condições equilibradas, a hemostasia é mantida por uma interação complexa entre fatores endoteliais, plaquetários e de coagulação. Situações que envolvem lesão e rompimento do revestimento endotelial estimulam a adesão, ativação e agregação de plaquetas, o que culmina na formação de trombos arteriais ou venosos. Nesse contexto, o tratamento antiplaquetário ocupa um papel de destaque no manejo de patologias resultantes desse processo, principalmente síndromes coronarianas agudas. O aumento da compreensão conceitual dos receptores, agonistas e antagonistas das cascatas fisiopatológicas envolvidas nesse processo permitiu o desenvolvimento de novos fármacos e o aprimoramento da terapia atual, exigindo um conhecimento completo do arsenal de agentes antiplaquetários no que se refere a indicação, dose, tempo de administração e duração do tratamento. O objetivo desta revisão é definir o papel das drogas antiplaquetárias no manejo da síndrome coronariana aguda, revisando aspectos já consolidados e que abordam questões atuais e ainda controversas sobre o assunto.

Palavras-chave: Síndrome coronariana aguda; Infarto do miocárdio; Agregação de plaquetas; Inibidores de plaquetas no sangue; Agentes antifibrinolíticos. 


\section{Introducción}

La cardiopatía isquémica es la principal causa de muerte y la pérdida de calidad de todo el mundo la vida (Vedanthan, Seligman, \& Fuster, 2014). En Brasil, se estima que esta relacionado con una tasa anual de aproximadamente 55 muertes por cada 100.000 habitantes (Piegas, Avezum, \& Guimarães, 2013). El síndrome coronario agudo (SCA), con su amplia gama de manifestaciones, adquiere un papel principal en estas estadísticas, y la revascularización miocárdica procedimientos y farmacoterapia antitrombótico son pilares fundamentales de su tratamiento.

La hemostasia es un proceso fisiológico mantenido a través de una compleja interacción entre factores endotelio, plaquetas y de la coagulación. En condiciones de emergencia y el desequilibrio, la hemostasia puede conducir a la formación de trombos arteriales o venosas, lo que resulta en la aparición de episodios coronarios agudos, eventos cerebrovasculares, o episodios de tromboembolismo. En este contexto, y junto con la terapia anticoagulante, antiplaquetas drogas se destacan en la gestión de estas condiciones.

Las situaciones que implican lesión y la interrupción del revestimiento endotelial estimulan proteínas plaquetarias adhesión a subendotelial matriz. Esta interacción activa señales intracelulares que promueven la liberación de adenosina difosfato (ADP), adrenalina, serotonina, trombina y tromboxano A2, potentes agonistas de la activación plaquetaria. Una vez que las plaquetas se activan, la glicoproteína Ilb-IIla complejos se unen al fibrinógeno para constituir la etapa final de la agregación plaquetaria y formación de trombos (Fuster \& Sweeny, 2011)

Los factores que activan este proceso y los receptores implicados en ella se han convertido en objetivos para el desarrollo de agentes antitrombóticos. Evidencia basada en medicina permitido a la llegada, la implementación y el perfeccionamiento del enfoque terapéutico actual. Sin embargo, mediante el aumento de la esperanza de vida de la población y la coexistencia de múltiples comorbilidades, que a menudo se encuentran escenarios clínicos en el que el riesgo de complicaciones isquémicas va de la mano con el riesgo de complicaciones hemorrágicas. Esto exige un conocimiento completo del arsenal de agentes antiplaquetarios en cuanto a su indicación, dosis y momento de la administración.

El objetivo de esta revisión es definir el papel de antiagregantes plaquetarios en la gestión de ACS, volviendo a visitar los aspectos que han sido ya consolidados y que abordan temas de actualidad y todavía controvertidos sobre el tema.

\section{Metodología}

Para el desarrollo de este proceso investigativo, se plantea como metodología la encaminada hacia una orientación científica particular que se encuentra determinada por la necesidad de indagar en forma precisa y coherente una situación, en tal sentido (Davila, 2015) define la metodología "como aquellos pasos previos que son seleccionados por el investigador para lograr resultados favorables que le ayuden a plantear nuevas ideas". (p.66)

Lo citado por el autor, lleva a entender que el desarrollo de la acción investigativa busca simplemente coordinar acciones enmarcadas en una revisión bibliográfica con el fin de complementar ideas previas relacionadas al tratamiento antiplaquetario en el síndrome coronario agudo a través de una revisión de literatura, para así finalmente elaborar un cuerpo de consideraciones generales que ayuden a ampliar el interés propuesto.

\section{Tipo de Investigación}

Dentro de toda práctica investigativa, se precisan acciones de carácter metodológico mediante las cuales, se logra conocer y

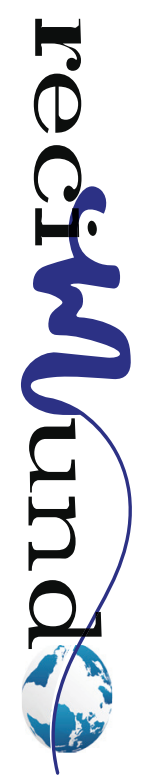


proyectar los eventos posibles que la determinan, así como las características que hacen del acto científico un proceso interactivo ajustado a una realidad posible de ser interpretada. En este sentido, se puede decir, que la presente investigación corresponde al tipo documental, definido por Castro (2016), "se ocupa del estudio de problemas planteados a nivel teórico, la información requerida para abordarlos se encuentra básicamente en materiales impresos, audiovisuales y $/ 0$ electrónicos". (p.41).

En consideración a esta definición, la orientación metodológica permitió la oportunidad de cumplir con una serie de actividades inherentes a la revisión y lectura de diversos documentos donde se encontraron ideas explicitas relacionadas con los tópicos encargados de identificar a cada característica insertada en el estudio. Por lo tanto, se realizaron continuas interpretaciones con el claro propósito de revisar aquellas apreciaciones o investigaciones propuestas por diferentes investigadores relacionadas con el tema de interés, para luego dar la respectiva argumentación a los planteamientos, en función a las necesidades encontradas en la indagación.

\section{Fuentes Documentales}

El análisis correspondiente a las características que predomina en el tema seleccionado, llevan a incluir diferentes fuentes documentales encargadas de darle el respectivo apoyo y en ese sentido cumplir con la valoración de los hechos a fin de generar nuevos criterios que sirven de referencia a otros procesos investigativos. Para (CASTRO, 2016) las fuentes documentales incorporadas en la investigación documental o bibliográfica, "representa la suma de materiales sistemáticos que son revisados en forma rigurosa y profunda para llegar a un análisis del fenómeno".(p.41). Por lo tanto, se procedió a cumplir con la realización de una lectura previa determinada para encontrar aquellos aspectos estrechamente vinculados con el tema, con el fin de explicar mediante un desarrollo las respectivas apreciaciones generales de importancia.

\section{Técnicas para la Recolección de la Infor- mación}

La conducción de la investigación para ser realizada en función a las particularidades que determinan a los estudios documentales, tiene como fin el desarrollo de un conjunto de acciones encargadas de llevar a la selección de técnicas estrechamente vinculadas con las características del estudio. En tal sentido, (Bolívar, 2015), refiere, que es "una técnica particular para aportar ayuda a los procedimientos de selección de las ideas primarias y secundarias". (p. 71).

Por ello, se procedió a la utilización del subrayado, resúmenes, fichaje, como parte básica para la revisión y selección de los documentos que presentan el contenido teórico. Es decir, que mediante la aplicación de estas técnicas se pudo llegar a recoger informaciones en cuanto a la revisión bibliográfica de los diversos elementos encargados de orientar el proceso de investigación. Tal como lo expresa, (Bolívar, 2015) "las técnicas documentales proporcionan las herramientas esenciales y determinantes para responder a los objetivos formulados y llegar a resultados efectivos" (p. 58). Es decir, para responder con eficiencia a las necesidades investigativas, se introdujeron como técnica de recolección el método inductivo, que hizo posible llevar a cabo una valoración de los hechos de forma particular para llegar a la explicación desde una visión general.

Asimismo, se emplearon las técnicas de análisis de información para la realización de la investigación que fue ejecutada bajo la dinámica de aplicar diversos elementos encargados de determinar el camino a recorrer por el estudio, según, (Bolívar, 2015) las técnicas de procesamiento de datos en los estudios documentales "son las encargadas de ofrecer al investigador la visión o pasos 
que debe cumplir durante su ejercicio, cada una de ellas debe estar en correspondencia con el nivel a emplear" (p. 123). Esto indica, que para llevar a cabo el procesamiento de los datos obtenidos una vez aplicado las técnicas seleccionadas, tales como: fichas de resumen, textual, registros descriptivos entre otros, los mismos se deben ajustar al nivel que ha sido seleccionado.

\section{Resultados}

\section{a) El ácido acetilsalicílico}

El ácido acetilsalicílico (ASA) a dosis bajas ciclooxigenasa selectivamente inhibe (COX) -1 , el establecimiento de la actividad antiplaquetaria, mientras que a altas dosis que inhibe la COX-1 y COX-2 con efectos anti-inflamatorios y analgésicos.

Este medicamento se absorbe rápidamente, alcanzando una concentración máxima en plasma 30 minutos después de la ingesta, en el caso de una formulación regular, y 4 horas después de la ingesta, en el caso de una formulación de liberación entérica ( $\mathrm{Pa}$ trono, Baigent, Hirsh, \& Roth, 2008)

Dado que los efectos secundarios gastrointestinales de aumento ASA en proporción a su dosis, una dosis de carga oral de 150$325 \mathrm{mg}$ (tragado, si una formulación regular, y masticó, si un uno enteral) se recomienda para ACS, o 80-150 mg por vía intravenosa en lugares en los que esta presentación está disponible, seguido de 81-100 mg como de mantenimiento. En el estudio pionero Segundo Estudio Internacional de Supervivencia del infarto (ISIS) -2, ASA promovido un significativo $23 \%$ de reducción en cardiovascular la mortalidad en comparación con placebo después de 5 semanas de tratamiento en pacientes con infarto agudo de miocardio (IAM) con elevación del segmento ST, con este efecto ser aditivo y sinérgico cuando se asocia con estreptoquinasa, la reducción de la tasa de eventos en un 42\% (ISIS-2 (Second International Study of Infarct Survival),
1988) Estas beneficios fueron corroborados por varios estudios posteriores y por la compilación de sus datos en grandes meta-análisis, convirtiéndose así en uno de los pilares de la farmacoterapia antitrombótico en los diferentes espectros de ACS.

Las situaciones que constituyen una contraindicación clara para ASA son raras, y incluyen sangrado digestivo principalmente activa y hipersensibilidad conocida (urticaria, broncoespasmo, o anafilaxia), aunque diferentes protocolos de desensibilización se han descrito lo que permite el uso crónico de estos medicamentos con eficacia y seguridad demostrada.

\section{B) P2Y12 receptor plaquetario antagonis- tas}

La asociación entre ASA y una P2Y12 antagonista del receptor, conocida como terapia antiplaquetaria dual, es la base del tratamiento de los pacientes con SCA y los sometidos a intervención coronaria percutánea $(\mathrm{PCl})$. La ticlopidina es una primera generación de tienopiridina derivado que, a pesar de su eficacia y debido a su hematológica (trombocitopenia, anemia aplástica, la púrpura trombocitopénica trombótica, neutropenia) y los efectos secundarios gastrointestinales, ha sido reemplazado rápidamente por clopidogrel (Steinhubl, Tan, Foody, \& Topol, 2009).

\section{b.1) Clopidogrel}

El clopidogrel es un agente de tienopiridina que forma irreversible bloquea el P2Y12 receptor. La inhibición de estado estacionario de la función plaquetaria con clopidogrel se consigue con una dosis de mantenimiento de $75 \mathrm{mg}$ después de 5-7 días desde el inicio del tratamiento, mientras que con la administración de 300 mg y 600 mg de dosis de carga, este efecto se alcanza en 6 y 2 horas, respectivamente, un aspecto relevante en el contexto de las emergencias médicas.

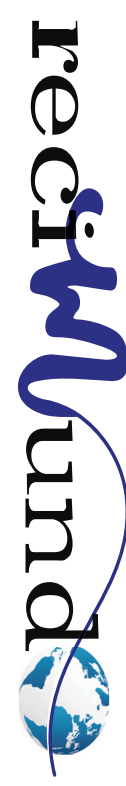


En el ensayo clínico de referencia clopidogrel en angina inestable para prevenir recurrentes Eventos (curación), 12.562 pacientes con sin elevación del ST IAM tratados con AAS fueron aleatorizados para recibir clopidogrel o placebo durante 3 a 12 meses (Yusuf, y otros, 2001) El grupo clopidogrel exhibió una reducción significativa del 20\% en el riesgo de enfermedades cardiovasculares muerte, preinfarto o accidente cerebrovascular en comparación con el grupo de placebo, con beneficios ya aparentes en las primeras horas después de la iniciación de la terapia e independiente de la estratificación invasiva. En el escenario de IAM con elevación del segmento ST, dos importantes estudios validados clopidogrel como una terapia adyuvante eficaz. Entre 3.491 pacientes asignados al azar a $300 \mathrm{mg}$ de clopidogrel y $75 \mathrm{mg}$ de clopidogrel mantenimiento versus placebo en el ensayo clínico de clopidogrel como adyuvante Terapia de reperfusión-Trombolisis en el Infarto de Miocardio (CLARITY-TIMI), de los cuales 28\% fueron sometidos a trombólisis, se observó 99.7 una reducción del 36\% en el riesgo de muerte, recurrente AMI, o ocluida la arteria del infarto relacionada en el momento de la angiografía (Sabatine, y otros, 2005). Por el contrario, en el estudio, Clopidogrel y Metoprolol en el Infarto de Miocardio Trial (COMMIT), entre 45.852 pacientes asignados al azar, con $50 \%$ de someterse a la trombólisis, la administración de una dosis diaria de 75 mg de clopidogrel durante un período medio de 28 días resultó en un 9 \% de reducción en las tasas de muerte, reinfarto o accidente cerebrovascular en comparación con el placebo (Chen, y otros, 2015).

Por lo tanto, el apoyo de los resultados de ensayos controlados aleatorios en ACS y por las características farmacodinámicas y farmacocinéticas de clopidogrel, se recomienda una dosis de carga de 600 mg para los pacientes en quien la estratificación del riesgo invasivo o que se predice son remitidos para la ICP primaria, y $300 \mathrm{mg}$ para pacientes se somete a terapia fibrinolítica.
Una excepción debe hacerse a pacientes con antecedentes de trombólisis que son de edad $\geq 75$ años, en los que la dosis de carga debe ser omitido. En ausencia de un claro beneficio del mantenimiento de clopidogrel en una dosis doble (150 mg), la dosis debe ser de $75 \mathrm{mg}$.

Sin embargo, clopidogrel presenta limitaciones y las necesidades no satisfechas, explica en parte por sus propiedades, tales como lento inicio y la retirada y el potencial de inhibición plaquetaria limitado. La variabilidad interindividual de la acción del fármaco es grande, con el porcentaje de pacientes que muestran inadecuada respuesta terapéutica estimada en hasta un 35\%. Los mecanismos involucrados son multifactoriales e incluyen interacciones de drogas (p.ej, inhibidores de la bomba de protones tales como omeprazol y esomeprazol), el medio ambiente y comorbilidades clínicos (p.ej, La adherencia a la terapia, tabaquismo, diabetes mellitus, obesidad, ACS), además de los polimorfismos genéticos, con énfasis en los alelos que determinan una pérdida de función CYP2C19.18 Se sabe que los pacientes con estas variantes están en mayor riesgo de trombosis del stent, la recurrencia de IAM y muerte (Holmes \& Dehmer, 2010) Sin embargo, aunque el tema sigue siendo objeto de escrutinio en los estudios en curso, la evidencia actualmente disponible no respalda la individualización de la terapia basada en los resultados de las pruebas genéticas o la medición de la función plaquetaria. Con eso, un vasto campo de la investigación se ha abierto con un tratamiento diferente propuestas, nuevo P2Y12 antagonistas de los receptores, y el bloqueo de nuevas dianas en la superficie de las plaquetas.

\section{b.2) Prasugrel}

Similar a clopidogrel, prasugrel es una tienopiridina de segunda generación que es un profármaco y requiere biotransformación para convertirse en un metabolito activo. Sin embargo, tiene un inicio más temprano de 
la acción, a unos 30 minutos después de la administración de una dosis de carga de 60 mg, además de una respuesta más predecible, debido a su amplia absorción, una necesidad de una única etapa de oxidación mediado por CYP3A4 y CYP2B6, conferir una mayor biodisponibilidad, mayores niveles de inhibición de las plaquetas, y menos variabilidad de la respuesta entre los pacientes.

En el ensayo para evaluar la mejoría en resultados terapéuticos mediante la optimización de plaquetas La inhibición con Prasugrel (TRITON) -TIMI 38 ensayo clínico, 13.608 pacientes ingresados por SCA con o sin elevación del segmento ST sometidos a ICP fueron aleatorizados para recibir una dosis de carga de $60 \mathrm{mg}$ de prasugrel y $10 \mathrm{mg}$ dosis de mantenimiento, o una dosis de carga de 300 mg de clopidogrel y 75 dosis de mantenimiento mg hasta por 15 meses (Wiviott, Braunwald, \& McCabe, 2007). En el tratamiento Prasugrel se demostró que era superior con una reducción significativa del 19\% en el riesgo de enfermedades cardiovasculares muerte, IAM o ictus no mortal, con una reducción del infarto de $24 \%$ y trombosis del stent de $52 \%$, con una beneficio aún mayor entre los pacientes con diabetes.

En consonancia con el aumento de la potencia de antiplaquetario prasugrel, el estudio encontró un aumento significativo del 32\% en el riesgo de hemorragia grave, incluso mortal y la vida hemorragia mortal. En los perfiles de algunos pacientes, la red beneficio clínico, que considera la eficacia anti-isquémica y la seguridad, era desfavorable para prasugrel, incluyendo pacientes con un ictus previo o ataque isquémico transitorio (TIA), con edades $\geq 75$ años, y con un peso corporal $<60 \mathrm{~kg}$. En las dos últimas situaciones, en caso de que haya una necesidad de prescribir el fármaco y una falta de alternativas terapéuticas, una dosis de mantenimiento de $5 \mathrm{mg}$ se recomienda. No hay indicios hasta la fecha en cuanto a la prescripción de prasugrel para pacientes con SCA que no se someten a ICP, o en un marco de tiempo que no sea después de que el conocimiento de la anatomía coronaria y la realización del procedimiento de intervención. En los pacientes con una indicación de cirugía de revascularización miocárdica, prasugrel idealmente debe interrumpirse durante 7 días.

\section{b.3) Ticagrelor}

La representante primera clínicamente disponible de la clase ciclopentiltriazolopirimidina, ticagrelor es un P2Y reversible12antagonista de los receptores, ejerciendo su efecto inhibidor a través de una unión no covalente a un sitio de unión de plaquetas diferente de ADP. A diferencia de la tienopiridina agentes, ticagrelor no es un profármaco y actúa inmediatamente después de la absorción oral. Exhibe, por lo tanto, un rápido inicio de la acción después de la ingestión de una dosis de carga de 180 mg y un pico de acción en 2 horas. Con una vida media de plasma de 8-12 horas, ticagrelor requiere una dosis de mantenimiento de $90 \mathrm{mg}$ cada 12 horas, y debido a que se une al receptor de una manera reversible, que tiene una terminación más temprana de la acción, alrededor de 2-3 días, a pesar de que el prospecto recomienda su suspensión durante 5 días antes de una intervención quirúrgica. Dado que el metabolismo de ticagrelor está mediado por CYP3A4 o CYP3A45, la administración concomitante de inductores o inhibidores del CYP3A4 fuerte durante su uso debe ser evitado (Bonello, Laine, Kipson, \& Mancini, 2014).

En el ensayo clínico de plaquetas La inhibición y los resultados del paciente (Platón), 18.624 pacientes que ofrecen todas las manifestaciones de ACS (con la excepción de aquellos con IAM con elevación del segmento ST que la trombolisis se sometieron a) fueron aleatorizados para recibir ticagrelor o clopidogrel durante 12 meses (Wallentin, Becker, \& Budaj, 2009). En el grupo de ticagrelor, hubo una disminución significativa reducción del 16\% en el riesgo de muerte cardiovascular, IAM o carrera. En un preespecificado análisis jerárquico, ticagrelorsola redujo la ocurrencia de muerte cardiovas- 
cular $(21 \%)$, AMI (16\%), y la trombosis definitiva stent (33\%), sin diferencia en la tasa de hemorragia grave o mortal. A pesar de la consistencia de los resultados obtenidos entre los diferentes subgrupos analizados, hubo una interacción entre los pacientes tratados en América del Norte y el resto del mundo, sin un claro beneficio de ticagrelor en el primer grupo.

El uso de altas dosis de mantenimiento de ASA ( $\geq 200$ mg) se cree que es una justificación probable para este hallazgo, lo que provocó una alerta para la prescripción de dosis <200 mg en el uso crónico.

El ticagrelor exhibe efectos pleiotrópicos no mediadas por su bloqueo de la P2Y12 receptor, incluyendo la inhibición de la captación de adenosina por los eritrocitos, lo que aumenta los niveles circulantes de adenosina. La adenosina es conocida por tener varias propiedades, incluyendo la vasodilatación coronaria, la reducción de la isquemia y lesión por reperfusión, la inhibición de las respuestas inflamatorias a condiciones de estrés, cronotrópico negativo y el efecto dromotropo, la reducción de glomerular la tasa de filtración, y la estimulación de las fibras vágales $\mathrm{C}$ en el livianos. Estos efectos están todavía bajo investigación y justificarían, entre los usuarios de ticagrelor, una mayor prevalencia de disnea y sin alteración del pulmonar.

Las pruebas de función, hace una pausa ventricular sin impacto clínico o requisito de un marcapasos definitivo, elevado de creatinina en suero y niveles de ácido úrico, así como una mayor estabilidad eléctrica y una reducción de las muertes súbitas arrítmicos, este último todavía en la especulación.

La Tabla 1 proporciona una recopilación de las principales indicaciones, dosis y recomendaciones con respecto a la prescripción de P2Y12 antagonistas de los receptores en SCA.

Tabla 1. Indicaciones de los antagonistas del receptor P2Y12 en síndromes coronarios agudos

\begin{tabular}{lccccc}
\hline Fármaco & Indicación & Dosis de carga & Dosis de mantenimiento & $\begin{array}{c}\text { Duración de } \\
\text { tratamiento }\end{array}$ & $\begin{array}{c}\text { Suspensión para } \\
\text { cirugía }\end{array}$ \\
\hline clopidogrel & $\begin{array}{c}\text { AMI con post-trombolisis ST } \\
\text { elevación }\end{array}$ & $300 \mathrm{mg} *$ & $75 \mathrm{mg} /$ día & 1 año & 5 dias \\
clopidogrel & $\begin{array}{c}\text { ACS sin elevación del ST } \\
\text { ICP primaria }\end{array}$ & $300-600 \mathrm{mg}$ & $75 \mathrm{mg} /$ día & 1 año & 5 dias \\
prasugrel & SCA tratados con ICP & $60 \mathrm{mg}$ & $10 \mathrm{mg} /$ día $\dagger$ & 1 año & 7 días \\
ticagrelor & ACS (excepto post-trombolisis) & $180 \mathrm{mg}$ & $90 \mathrm{mg}$ cada 12 horas & 1 año & $3-5$ días \\
\hline
\end{tabular}

AMI: infarto agudo de miocardio; ACS: síndrome coronario agudo; ICP: intervención coronaria percutánea. * La dosis de carga se omite si edades $\geq 75$ años. + Dosis de mantenimiento de $5 \mathrm{mg}$ si el peso $<60 \mathrm{~kg}$ o edad $\geq 75$ años.

Fuente: Los Autores 2020 
Tabla 2. ropiedades farmacológicas de inhibidores de la glucoproteína lib-IIla

\begin{tabular}{lcc} 
& Abciximab & tirofibán \\
\hline Estructura & anticuerpo monoclonal & antagonista no peptídico \\
Peso molecular & $48 \mathrm{kDa}$ & $<1 \mathrm{kDa}$ \\
Comienzo & Rápido & Rápido \\
Reversibilidad & Lenta $(12$ horas $)$ & Fast $(2$ horas $)$ \\
Media vida & $10-30$ minutos & 2 horas \\
Excreción & Desconocido & Renal $(40-70 \%)$ y biliar \\
dosis de carga & $0,25 \mathrm{mg} / \mathrm{kg}$ & $25 \mathrm{mg} / \mathrm{kg}$ \\
dosis de mantenimiento & $0,125 \mathrm{mg} / \mathrm{kg} / \mathrm{min}$ durante 12 horas & $0,15 \mathrm{~g} / \mathrm{kg} / \mathrm{min}$ durante $18 \mathrm{horas}$ \\
El ajuste de dosis & No & $\downarrow 50 \%$ si el aclaramiento de $\leq 30 \mathrm{ml} / \mathrm{min}$ \\
\hline
\end{tabular}

Fuente: Los Autores 2020

\section{c) Inhibidores de la glucoproteína Illb-Illa}

Los inhibidores de la glicoproteína Ilb-IIla aprobados para uso clínico - abciximab, tirofibán y eptifibatida - son antagonistas plaquetarios potentes de administración parenteral, de los cuales sólo los dos primeros son disponibles comercialmente en Brasil. Las características farmacológicas de estos agentes se resumen en la Tabla 2.

Abciximab es un anticuerpo monoclonal con una alta afinidad para la glicoproteína receptores IIb-IIIa. Después de una dosis de carga de 0,25 mg / kg, más del $80 \%$ de los receptores están bloqueados, con una reducción de la agregación plaquetaria en respuesta a la estimulación ADP a menos del 20\% del valor de línea de base. Esta inhibición es mantenido por una infusión continua de abciximab a una dosis de 0,125 mg $/ \mathrm{kg} / \mathrm{min}$, hasta un máximo de $10 \mathrm{~g} / \mathrm{min}$. La trombocitopenia se informa en el 1-4\% de los pacientes tratados con el fármaco, comúnmente detectado dentro de las primeras 24 horas y hasta 2 horas después de la iniciación de su administración. Esto se cree que es una complicación mediada por anticuerpos, con una inversión en la mayoría de los casos después de su suspensión y un requisito ocasional de transfusión de plaquetas (Moussa \& Bennet, 2006)

Tirofibán es un derivado de tirosina no péptido que actúa a través de un bloqueo reversible y selectiva del receptor IIb-IIla de glicoproteína, evitando su unión a fibrinógeno y el factor de von Willebrand. Con la excreción renal y biliar, tirofiban tiene una media de plasma vida de 1.5-2 horas, con el requisito de un ajuste de dosis en pacientes con insuficiencia renal y creatinina aclaramiento de $\leq 30 \mathrm{ml} / \mathrm{min}$, pero no en los casos de enfermedad hepática. Cuando se administra a una dosis de carga de $25 \mathrm{mg} / \mathrm{kg}$, tirofibán exhibe una eficacia similar a la de abciximab. Los casos de trombocitopenia grave, aunque poco frecuente y reversible, también se han reportado. La aparición de trombocitopenia grave se atribuye a un mediado inmune fenómeno, ya que la unión de tirofibán promueve un cambio conformacional en el receptor, con la generación de anticuerpos contra el nuevo epítopo expuesto.

La evidencia de que se ha validado el uso de inhibidores de la glucoproteína (GPI) IIb-IIIa en la ICP, dada la capacidad de estos agentes para reducir periprocedimental IAM y la necesidad de revascularización

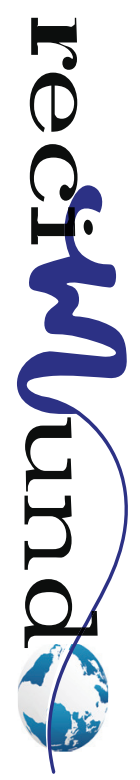


urgente, se originó principalmente de estudios clínicos realizados antes de la llegada de la tienopiridina agentes y la implantación del stent rutina. En la era moderna de la cardiología intervencionista, los meta-análisis se han realizado estudios para evaluar el papel real que desempeñan estos agentes como terapia adyuvante para el $\mathrm{PCl}$.

En el tratamiento de la no elevación del ST ACS, un meta-análisis que implica 31,402 pacientes demostró una leve pero un beneficio significativo en la reducción de muertes y IAM a los 30 días, favoreciendo el uso de inhibidores de la glucoproteína IIb-IIla, especialmente entre los pacientes considerados de alto riesgo (p.ej, Los pacientes con marcadores de necrosis miocárdica positivos, aumento de la carga trombótica, o lesiones complejas). Un metaanálisis que participan siete ensayos aleatorios y 19.929 pacientes evaluó la temporización más adecuada para la administración de inhibidores de la glucoproteína Ilb-IIla (De Luca, Navarese, \& Cassetti, 2011).

Una estrategia de infusión temprano antes de la admisión del paciente al laboratorio de cateterización no fue capaz de reducir la incidencia de la mortalidad a los 30 días o AMI recurrente. Sin embargo, se asocia con una mayor tasa de hemorragias graves.

Así, los inhibidores IIb-IIla desempeñan un papel importante como terapia adyuvante en $\mathrm{PCl}$, pero la prescripción de estos agentes se limita a las situaciones no programados, especialmente en el laboratorio de cateterismo, con énfasis en los pacientes con alto riesgo no del segmento ST elevación ACS y sin tratamiento previo con P2Y12 inhibidores del receptor, de la presencia de complicaciones trombóticas durante la ICP (abrupta oclusión de los vasos, lento flujo epicárdico, la disección coronaria, rama lateral oclusión) a IAM con elevación del segmento ST en pacientes de alto riesgo o en presencia de un aumento del trombótica carga. Tras la consideración de estos agentes, el perfil de riesgode los pacientes se debe considerar en relación con la ocurrencia de eventos hemorrágicos, que son conocidos promotores de aumento de la morbilidad y la mortalidad.

Aunque el uso de inhibidores de la glicoproteína Ilb-IIla tiene sin la influencia sobre la eficacia de la nueva antiplaquetario oral fármacos (ticagrelor, prasugrel) en el tratamiento de ACS, se requiere más investigación en cuanto a su uso en pacientes tratados con el nuevo P2Y12 inhibidores de los receptores, o aquellos con variantes CYP2C19 genéticos o respuesta de baja clopidogrel, o con respecto a la opción de reducir o suprimir su dosis de mantenimiento.

\section{d) El pretratamiento con P2Y12 antago- nistas de los receptores}

En la fase aguda de ACS, un escenario caracterizado por un estado protrombótico y la activación de plaquetas intenso, dual aglutinación antiplaquetario representa un importante paso terapéutico, y es intuitivo para asumir que una acción potente y principios farmacológica es capaz de conferir eficacia anti-isquémica, reduciendo trombótica eventos (infarto periprocedimiento, trombosis del stent, reoclusión coronaria), especialmente entre los pacientes sometidos a la estratificación del riesgo invasivo y $\mathrm{PCl}$. Sin embargo, contrapuntos a un tratamiento previo con P2Y12 antagonistas de los receptores incluyen el aumento de la prevalencia de complicaciones hemorrágicas con la terapia antitrombótica, así como un posible retraso en la aplicación de tratamiento quirúrgico en pacientes con enfermedad de múltiples vasos y la indicación de la revascularización, la prolongación de la duración de hospitalización y aumentar el riesgo de hemorragia relacionado con la cirugía. Aún así, hay escasas pruebas de los ensayos clínicos aleatorios con la casuística adecuada y el poder estadístico para obtener una respuesta clara y definitiva a este tema.

El juicio de clopidogrel para la reducción de 
Ios eventos durante la observación (CREDO) es uno de los pioneros estudios que sugieren un beneficio de principios antiplaquetaria dual terapia inició inmediatamente después del diagnóstico. Este ensayo asignó al azar a 2.116 pacientes, incluyendo $66 \%$ diagnosticados con SCA (Wiviott, Braunwald, \& McCabe, 2007). El objetivo del ensayo fue evaluar el beneficio de una dosis de carga de $300 \mathrm{mg}$ de clopidogrel 3-24 horas antes de la ICP y mantenimiento de la doble terapia durante 12 meses. El pretratamiento con clopidogrel se asoció con una reducción no significativa del 18\% en el riesgo de muerte, IAM o revascularización urgente en 28 días. Sin embargo, en un análisis de subgrupos preespecificado, una reducción significativa del $38,6 \%$ en el resultado primario se observó en los pacientes cuya terapia se inició 6 horas antes de la ICP, la base conceptual para la adopción de esta estrategia.

En la era de la nueva y potente P2Y12 inhibidores de los receptores, el estudio Comparación de Prasugrel en el momento de coronaria percutánea intervención o como pretratamiento en el momento del diagnóstico en pacientes con no elevación del ST infarto de miocardio (ACCOAST) asignó al azar 4.033 pacientes con SCA sin elevación del segmento ST para recibir una 30 $\mathrm{mg}$ dosis de prasugrel en el momento del diagnóstico (pretratamiento), además de 30 mg en el caso de $\mathrm{PCl}$, en comparación con $60 \mathrm{mg}$ inmediatamente antes del procedimiento (Montalescot, Bolognese, \& Dudek, 2013). El primario punto final de eficacia, compuesto de muerte cardiovascular, IAM, ictus, revascularización urgente, o el uso no planificado de inhibidores de la glucoproteína IIb-IIIa a los 7 días no fue diferente entre los grupos, mientras que el resultado de seguridad, severa ocurrencia de sangrado por la clasificación TIMI, era casi duplicará entre aquellos tratados previamente con prasugrel, con su uso en tales circunstancias se desanimó.

\section{e) La duración óptima de la terapia anti- plaquetaria dual}

Se recomienda la inhibición plaquetaria doble durante un periodo de 1 año para los pacientes diagnosticados de SCA, especialmente aquellos que se sometieron a cirugía de revascularización miocárdica percutánea, con independencia del tipo de stent; esta recomendación se basa en los resultados de ensayos clínicos aleatorios tales como CURE y, más recientemente, TRITON-TIMI 38 y Platón, indicando una beneficio temprana y continua de la terapia antiplaquetaria dual de más de 12 meses de tratamiento (Montalescot, van't Hof, \& Lapostolle, Prehospital ticagrelor in ST-segment elevation myocardial infarction., 2014).

Un tema reciente de la discusión es el mantenimiento de la terapia antiplaquetaria dual para más de 12 meses entre los individuos sometidos a ICP. Un meta-análisis que abarca seis ensayos clínicos aleatorios y 33,435 pacientes con una historia previa de IAM mostró que la extensión de la doble antiagregación después de 1 año promueve una reducción significativa de eventos cardiovasculares combinados comparación con la monoterapia con AAS, lo que demuestra una reducción aislada de muerte cardiovascular, IAM, accidente cerebrovascular y trombosis del stent a expensas de una mayor incidencia de hemorragias graves (Steinhubl, Tan, Foody, \& Topol, 2009).

\section{Conclusión}

La farmacoterapia antiplaquetaria juega un papel decisivo en la gestión clínica y invasivo de ACS. El mayor conocimiento de la fisiopatología de las cascadas que participan en este proceso ha permitido la llegada de los tipos más eficaces de tratamiento, a menudo asociados con un mayor riesgo de sangrado. Sin embargo, la seguridad de cualquier prescripción es de suma importancia en la práctica contemporánea, ya que las complicaciones hemorrágicas conllevan un alto riesgo de morbilidad y mortalidad. Muchos estudios de investigación en

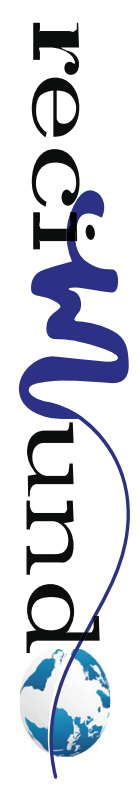


curso siguen esta corriente tema controvertido, y están sujetas a cambios regulatorios constantes enfoques. Por lo tanto, siempre por nosotros mismos guiar por el sentido común, individualizado, y la estimación de los riesgos y beneficios, que puede directamente interferir en el pronóstico y la evolución de los pacientes.

\section{Bibliografía}

Bolívar, J. (2015). Investigación Documental. México: Pax.

Bonello, L., Laine, M., Kipson, N., \& Mancini, J. (2014). Ticagrelor increases adenosine plasma concentration in patients with acute coronary syndrome. J Am Coll Cardiol, 872-7.

Castro, J. (2016). Técnicas Documentales. México: Limusa.

Chen, Z., Jiang, L., Chen, Y., Peto, R., Collins, R., \& Jiang, L. (2015). Addition of clopidogrel to aspirin in 45,852 patients with acute myocardial infarction: randomised placebo-controlled trial. Lancet, 1607-21.

Davila, A. (2015). Concepto de terminos cientificos. Caracas: Oasis.

De Luca, G., Navarese, E., \& Cassetti, E. (2011). Meta-analysis of randomized trials of glycoprotein llb/ IIla inhibitors in high-risk acute coronary syndromes patients undergoing invasive strategy. . Am J Cardiol., 198-203.

Fuster, V., \& Sweeny, J. (2011). Aspirin: a historical and contemporary therapeutic overview. . Circulation., 768-78.

Holmes, D. J., \& Dehmer, G. (2010). ACCF/AHA clopidogrel clinical alert: approaches to the FDA "boxed warning: " a report of the American College of Cardiology Foundation Task Force on Clinical Expert Consensus Documents and and the American Heart Association. . J Am Coll Cardiol., 56.

ISIS-2 (Second International Study of Infarct Survival). (1988). Collaborative Group. Randomised trial of intravenous streptokinase, oral aspirin, both, or neither among 17187 cases of suspected acute myocardial infarction. Lancet, 349-60.

Montalescot, G., Bolognese, L., \& Dudek, D. (2013). ACCOAST Investigators. Pretreatment with prasugrel in non-ST-segment elevation acute coronary syndromes. N Engl J Med., 999-1010.
Montalescot, G., van't Hof, A., \& Lapostolle, F. (2014). Prehospital ticagrelor in ST-segment elevation myocardial infarction. N Engl J Med. , 1016-27.

Moussa, S., \& Bennet, J. (2006). Platelets in health and disease: platelet GP IIb/Illa structure and function: recent advances in antiplatelet therapy. . Drugs Future, 1141-54.

Patrono, C., Baigent, C., Hirsh, J., \& Roth, G. (2008). Antiplatelet drugs: American College of Chest Physicians Evidence-Based Clinical Practice Guidelines. Chest, 199S-233S.

Piegas, L., Avezum, A., \& Guimarães, H. (2013). Acute coronary syndrome behavior: results of a Brazilian registry. Arq Bras Cardiol, 502-10.

Sabatine, M., Cannon, C., Gibson, C., López-Sendón, J., Montalescot, G., \& Theroux, P. (2005). CLARITY-TIMI 28 Investigators. Addition of clopidogrel to aspirin and fibrinolytic therapy for myocardial infarction with ST-segment elevation. N Engl J Med., 352.

Steinhubl, S., Tan, W., Foody, J., \& Topol, E. (2009). Incidence and clinical course of thrombotic thrombocytopenic purpura due to ticlopidine following coronary stenting. EPISTENT Investigators. Evaluation of Platelet IIb/ IIla Inhibitor for Stenting. JAMA, 3-25.

Vedanthan, R., Seligman, B., \& Fuster, V. (2014). Global perspective on acute coronary syndrome: a burden on the young and poor. Circ Res, 1959-75.

Wallentin, L., Becker, R., \& Budaj, A. (2009). Ticagrelor versus clopidogrel in patients with acute coronary syndromes. N Engl J Med, 1045-57.

Wiviott, S., Braunwald, E., \& McCabe, C. (2007). Prasugrel versus clopidogrel in patients with acute coronary syndrome. N Engl J Med, 2001-15.

Yusuf, S., Zhao, F., Metha, S., Chrolavicius, S., Toçnoni, G., \& Fox, K. (2001). The clopidogrel in unstable angina to prevent recurrent events trial investigators. Effects of clopidogrel in addition to aspirin in patients with acute coronary syndromes without ST-segment elevation. N Engl J Med, 494-502. 


\section{CITAR ESTE ARTICULO:}

Salinas Romero, J., Arana Alcivar, Y., Sánchez León, D., \& Guachi Criollo, A. (2020). Agentes antiagregantes en los síndromes coronarios agudos. REClMUNDO, 4(1), 71-83. doi:10.26820/recimundo/4.(1).enero.2020.71-83 